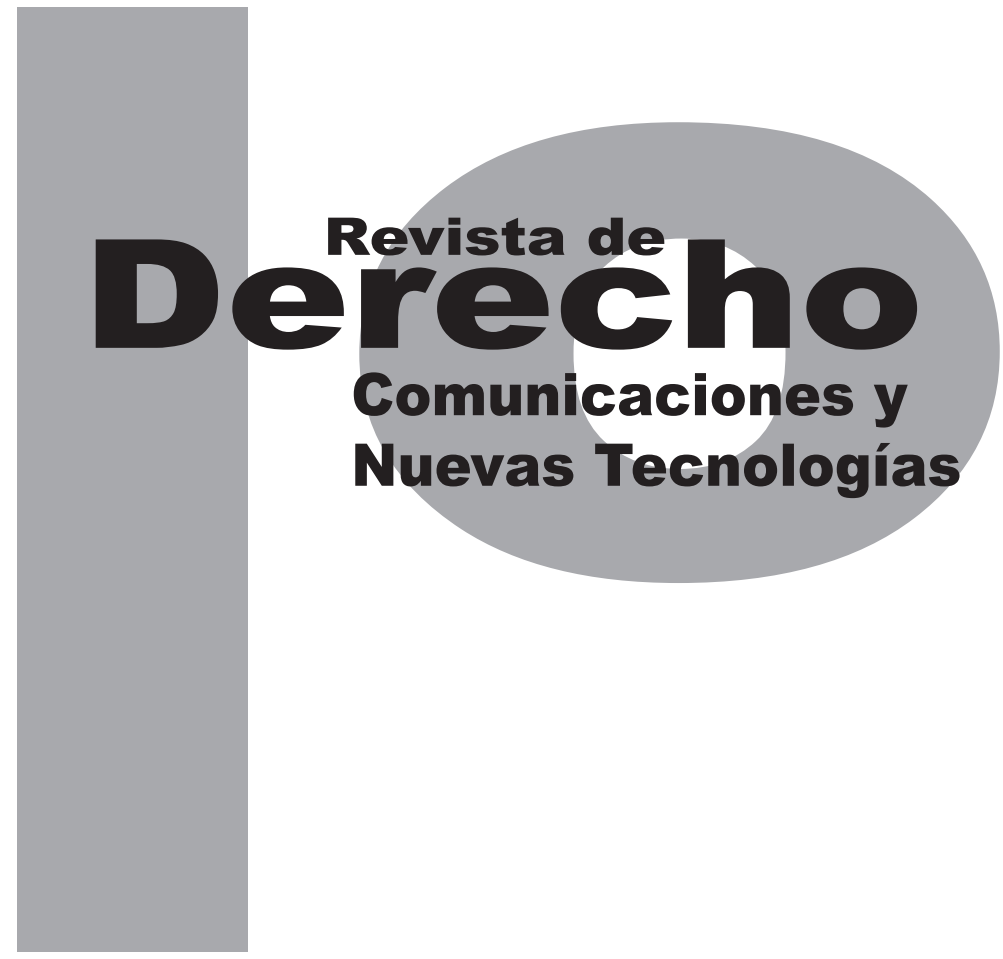

\title{
Comercio electrónico, Online Dispute Resolution Y DESARROLLO
}

\author{
NURIA GONZÁLEZ MARTíN \\ MARía Mercedes Albornoz \\ Artículo de revisión \\ DOI: http://dx.doi.org/10.15425/redecom.12.2014.12
}

Universidad de los Andes

Facultad de Derecho

Revista de Derecho, Comunicaciones y Nuevas Tecnologías

No. 12, Julio - Diciembre de 2014. ISSN 1909-7786 


\title{
Comercio electrónico, Online Dispute Resolution y desarrollo
}

\section{Resumen}

El presente artículo versa sobre las relaciones entre comercio electrónico y desarrollo. Adoptando una perspectiva analítica y reflexiva, aborda en primer lugar el comercio electrónico como propulsor del desarrollo y luego la resolución electrónica de disputas (ODR) como herramienta para fortalecer la seguridad jurídica en el ambiente electrónico. Concluye que el oDR, al aumentar la confianza en el comercio electrónico, y el comercio electrónico, al favorecer el crecimiento de la economía digital, pueden tener un impacto altamente positivo en el desarrollo de los países latinoamericanos. Si bien el comercio electrónico tiene grandes posibilidades de expansión en la región, para que despliegue todo su potencial aún se requiere fortalecer la seguridad jurídica en el ambiente virtual. Y a tal fin, el uso de un mecanismo de ODR -resultado de la combinación de los métodos alternativos de solución de controversias (MAsc) con las tecnologías de la información y de la comunicación (TIC) - escogido por las partes es una herramienta sumamente apropiada.

Palabras clave: comercio electrónico, tecnologías de la información y de la comunicación, desarrollo, resolución electrónica de disputas, medios alternativos de solución de controversias, seguridad jurídica.

\section{E-commerce, Online Dispute Resolution and development}

\begin{abstract}
The present article addresses the relationships between e-commerce and development. Adopting an analytical and reflective perspective, it copes first of all with e-commerce as promoter of development, and later with oDR as a tool to reinforce legal certainty in cyberspace. It concludes that ODR, by improving user's trust in e-commerce, and e-commerce itself, fostering the growth of digital economy, may together have a highly positive impact on the development of Latin American countries. Although e-commerce has great expansion possibilities in the region, in order for it to realize its full potential, legal certainty needs to be reinforced within the virtual realm. To this end, the use of an oDR mechanism (a result of combining ADR and ICT together) when chosen by the parties involved, becomes a very suitable tool.
\end{abstract}

Keywords: electronic commerce, information and communication technologies, development, Online Dispute Resolution, Alternative Dispute Resolution, legal certainty.

\section{Comércio eletrônico, Online Dispute Resolution e desenvolvimento}

\section{Resumo}

O presente artigo versa sobre as relações entre comércio eletrônico, online dispute resolution (ODR) e desenvolvimento. Adotando uma perspectiva analítica e reflexiva, aborda em primeiro lugar o comércio eletrônico como propulsor do desenvolvimento e depois o ODR como ferramenta para fortalecer a segurança jurídica no ambiente eletrônico. Conclui que o ODR, ao aumentar a confiança no comércio eletrônico, e este último, ao favorecer o crescimento da economia digital, podem ter um impacto altamente positivo no desenvolvimento dos países latino-americanos. Se bem que o comércio eletrônico tem grandes possibilidades de expansão na região, mas para que utilize todo o seu potencial ainda se requer fortalecer a segurançajurídica no ambiente virtual. E a tal fim o uso de ODR -resultado da combinação dos mecanismos alternativos de solução de controvérsias (MASC) com as tecnologias da informação e comunicação (TICS)escolhido pelas partes é uma ferramenta sumamente apropriada.

Palavras-chave: comércio eletrônico, tecnologias da informação e da comunicação, desenvolvimento, resolução eletrônica de disputas, meios alternativos de solução de controvérsias, segurança jurídica. 


\title{
Comercio electrónico, Online Dispute Resolution y desarrollo*
}

\author{
Nuria González Martín** \\ María Mercedes Albornoz ${ }^{* * *}$
}

\section{SUMARIO}

Introducción - I. COMERCIO ELECTRÓNICO COMO PROPULSOR DEL DESARROLLO - A. Concepto de comercio electrónico - B. Función del comercio electrónico con respecto al desarrollo - II. ODR COMO HERRAMIENTA PARA FORTALECER LA SEGURIDAD JURÍDICA EN EL AMBIENTE ELECTRÓNICO - A. La seguridad jurídica en el ambiente electrónico - B. Concepto de oDR y su función con respecto a la seguridad jurídica - III. CONCLUSIONES - Referencias.

* Cómo citar este artículo: González Martín, N. y Albornoz, M. M. (Diciembre, 2014). Comercio electrónico, Online Dispute Resolution y desarrollo. Revista de Derecho, Comunicaciones y Nuevas Tecnologías, 12. Universidad de los Andes (Colombia).

** Doctora en Derecho Internacional Privado, Universidad Pablo de Olavide, Sevilla, (España). DEA (LLM) en Derecho, Universidad de Sevilla. Investigadora del Instituto de Investigaciones Jurídicas, Universidad Nacional Autónoma de México (UNAM). Directora del Boletín Mexicano de Derecho Comparado. Académica visitante en Stanford Law School (2012-2015). Correo: nuria.gonzalez68@gmail.com.

*** Abogada egresada de la Universidad Nacional del Litoral (Argentina). DEA (LLM) y Doctora en Derecho Internacional Privado, Université de Paris II, Panthéon-Assas (Francia). Profesora investigadora de la División de Estudios Jurídicos del Centro de Investigación y Docencia Económicas, CIDE (México). Correo: mercedes.albornoz@cide.edu. 


\section{Introducción}

Los países en desarrollo, categoría que incluye a los países latinoamericanos considerados en su conjunto, ${ }^{1}$ continúan rezagados con respecto a los países más avanzados del planeta. Incluso si se toma en cuenta la heterogeneidad existente al interior de América Latina, la brecha que los separa de los países económicamente más favorecidos aún dista de cerrarse. Sin embargo, los avances registrados durante 2013 en varios países del Sur, entre los cuales se destacan en nuestra región Brasil como gran economía y Chile como economía más pequeña, han permitido que se hable de un "ascenso del Sur" (PNUD, 2013). Creemos que es necesario mantener ese impulso ascendente, propiciar su propagación a todos los países latinoamericanos y, sobre todo, procurar que el progreso humano sea sostenido ${ }^{2}$ en el tiempo.

Las tecnologías de la información y de la comunicación (TIC) son -entre otros elementos, por supuesto- un factor coadyuvante al desarrollo de los pueblos. En este artículo sostenemos que también lo son el comercio electrónico que aquéllas permiten y la resolución electrónica de disputas (Online Dispute Resolution -ODR),

1 Si bien en el sistema de las Naciones Unidas la distinción entre países desarrollados y países en desarrollo no responde a nomenclatura oficial alguna, en la práctica de dicho organismo internacional se considera que "las Américas", excepto América del Norte (Canadá y Estados Unidos), son una región en desarrollo (UNdata, 2013).

2 En este sentido, destacamos que el Informe sobre Desarrollo Humano 2014 del PNUD se enfoca justamente en cómo sostener el progreso humano, reducir vulnerabilidades y construir resiliencia. Resumen en español disponible en: http://hdr.undp.org/sites/default/files/hdr14summary-es.pdf. Texto completo en inglés disponible en: http://hdr. undp.org/sites/default/files/hdr14-report-en-1.pdf. Consultados el 5 de agosto de 2014 en tanto esta última estimula la confianza en el comercio electrónico. En efecto, los constantes y vertiginosos avances de las Tic, así como su cada vez mayor difusión entre la población, ${ }^{3}$ crean un entorno propicio para la expansión de la compraventa de bienes y servicios llevada a cabo a través de Internet o de diversos dispositivos móviles. Entendemos que, hoy por hoy, la relevancia de la que goza el comercio electrónico en pleno siglo XXI está totalmente fuera de discusión.

De acuerdo con el informe The World in 2014. ICT Facts and Figures, de la Unión Internacional de Telecomunicaciones (ITU, por sus siglas en inglés), para finales de 2014 el número de usuarios de Internet en el mundo será de 3000 millones de personas, lo que equivale a prácticamente al $40 \%$ de la población mundial. De entre ellos, dos tercios pertenecen a países en desarrollo, donde el número de usuarios de Internet se habrá duplicado en un período de cinco años, pasando de 974 millones en 2009 a 1900 millones en 2014. En los países en desarrollo, solo el 32\% de la población está en línea, en comparación con el $78 \%$ de la población de los países desarrollados. Asimismo, de los 4000 millones de personas en todo el mundo que no son usuarios de Internet, el 90\% pertenece a países en desarrollo. En cuanto a América Latina en particular, si bien fue la región que regis-

3 Tanto se han expandido en la población mundial los avances de las TIC, que Zygmunt Bauman, en entrevista concedida al diario Clarín, de Buenos Aires, afirma: "Hoy vivimos simultáneamente en dos mundos paralelos y diferentes. Uno, creado por la tecnología online, nos permite transcurrir horas frente a una pantalla. Por otro lado tenemos una vida normal... en el mundo que, en oposición al mundo online, llamo offline" Artusa (2014). 
tró un mayor crecimiento de usuarios de Internet (12\%) de marzo de 2012 a marzo de 2013 (comScore, 2013, p. 6), continúa rezagada en lo atinente a nuevas tecnologías (Cepal, 2013, p. 46). Una de las dificultades para el desarrollo de las TIC y de Internet en la región es la heterogeneidad entre sus países. ${ }^{4}$ Asimismo, en tanto algunos países de la región tienden a reducir la brecha digital que los separa de los países desarrollados, ${ }^{5}$ otros países de la misma región tienden a aumentarla y todos se desenvuelven a diverso ritmo (Cepal, 2013, p. 20).

Por otro lado, a medida que el número de abonados a servicios móviles celulares se va aproximando a las cifras de la población mundial, va disminuyendo el crecimiento de la cantidad de abonados a tales servicios (ITU, 2013). Para finales de 2014 se estima que la tasa de penetración de servicios móviles celulares a nivel mundial será del 96\%, alcanzando el 90\% en los países en desarrollo y el $121 \%$ en los países desarrollados (ITU, 2014). Asimismo, de los 6900 millones de suscripciones a este tipo de servicios que se espera que haya en el mundo al finalizar 2014, el 78\%, es decir, más de las tres cuartas partes del total, corresponderá a países en desarrollo (ITU, 2014). En América Latina, la tasa de penetración de telefonía móvil continúa en ascenso: del 76\% en 2007 ha pasado al

$4 \quad$ En el índice de uso de TIC, la diferencia entre los tres países de mejor desempeño y los tres países de peor desempeño superaba en 2011 el $300 \%$ (Cepal, 2013, p. 19).

5 Esto se refleja en la reducción global regional de la brecha digital con los países desarrollados. Sin embargo, a su vez genera una mayor heterogeneidad intrarregional (Cepal, 2013, p. 22).
$106 \%$ en 2011 y se espera que alcance el $126 \%$ en 2015 (Statista, 2014).

Si bien el servicio de banda ancha móvil sigue creciendo -será de 2300 millones a finales de 2014- (ITU, 2014), un dato que merece ser destacado es que en los países en desarrollo su costo es mucho mayor que en los países más avanzados, aunque se trata de un costo inferior al de los servicios de banda ancha fija (ITU, 2013).

En cuanto al comercio electrónico, se prevé que en 2014 el gasto mundial aumentará un 90\% (America Retail, 2013). América Latina ha tenido un progreso significativo en este ámbito durante los últimos años ${ }^{6}$ y se estima que en 2014 el crecimiento del comercio electrónico será del 204\%, ubicándose por delante de Europa del Este -180\%- y de Asia-Pacífico -71\% - (America Retail, 2013).

Inclusive en los países en desarrollo, el comercio electrónico llegó, en efecto, para quedarse. Aunque no creemos que vaya a desterrar totalmente las transacciones sin conexión (offline), ni que la desaparición absoluta de estas del escenario de la vida cotidiana sea siquiera deseable, lo cierto es que el potencial de crecimiento del comercio electrónico en América Latina es enorme. Y que impulsarlo habrá de contribuir a incrementar el desarrollo de los países de nuestra región.

\footnotetext{
6 Las visitas de internautas latinoamericanos a sitios comerciales fueron del $76 \%$ del total de los usuarios, llegando al $91 \%$ en Brasil, $80 \%$ en Argentina y $72 \%$ en Chile (Cepal, 2013, p. 13).
} 
Ahora bien, el ambiente virtual en el cual se desenvuelve el comercio electrónico es susceptible de generar falta de confianza, especialmente en cabeza del consumidor electrónico. Dicha sensación, que se exacerba cuando la transacción presenta elementos de extranjería -es decir, cuando es transfronteriza o internacional-, puede responder a distintos motivos. Uno de ellos es la incertidumbre de qué hacer o adónde recurrir si el vendedor o proveedor de un producto o un servicio en línea incumple total o parcialmente sus obligaciones, aunada a la gran preocupación por los desproporcionados costos que implicaría un eventual litigio en el extranjero. Es aquí donde entran en escena los novedosos mecanismos de Online Dispute Resolution (ODR), surgidos de la combinación del Alternative Dispute Resolution (ADR) y las modernas TIC. EI ODR constituye una herramienta que cuenta con una amplia capacidad para contribuir a apuntalar la seguridad jurídica en el entorno electrónico.

A continuación haremos un análisis del comercio electrónico como propulsor del desarrollo (I) y del ODR como herramienta para fortalecer la seguridad jurídica en el ambiente electrónico (II) que, indirectamente, podrá favorecer el desarrollo de los países de nuestra América Latina.

\section{COMERCIO ELECTRÓNICO COMO PROPULSOR DEL DESARROLLO}

A fin de concentrarnos en la función que el comercio electrónico puede desempeñar con respecto al desarrollo de los países latinoamerica- nos $(B)$, es propicio previamente aproximarnos al concepto de comercio electrónico $(A)$.

\section{A. Concepto de comercio electrónico}

En los últimos años, de la mano de las Tıc, el comercio electrónico ha adquirido una importancia central en la economía mundial y, específicamente, en el ámbito de la economía digital. ${ }^{7} \mathrm{El}$ intercambio de información ya no es físico sino que se efectúa por medios digitales, viéndose "reducido a bits almacenados en computadoras y circulando a través de redes" (Tapscott, 1997, p. 6).

Son muchos los elementos a considerar cuando se intenta construir una definición de "comercio electrónico". De esta manera, y sin ánimo de exhaustividad, sería factible adoptar una perspectiva amplia o una restringida en cuanto al medio empleado para expresar el consentimiento contractual, que podría ser cualquier medio electrónico, o bien únicamente Internet. Lo mismo sucedería con respecto al carácter de las redes en las que el comercio electrónico se lleva a cabo: una postura amplia permitiría abarcar tanto las transacciones realizadas en redes abiertas (Internet) como en redes cerradas (Intranet), mientras que una concepción restringida podría limitarse a uno u otro tipo de red. Si se sigue un enfoque amplio, bastaría para considerar electrónico cierto contrato el hecho de que este se haya perfeccionado en línea; en cambio,

"La economía digital está constituida por la infraestructura de telecomunicaciones, las industrias TIC (software, hardware y servicios TIC) y la red de actividades económicas y sociales facilitadas por Internet, la computación en la nube y las redes móviles, las sociales y de sensores remotos" (Cepal, 2013, p. 9). 
siguiendo una concepción más restringida, se exigiría, adicionalmente, que el cumplimiento de todas las prestaciones contractuales 0 al menos de alguna de ellas se efectúe en el ambiente virtual. Asimismo, podría incluirse en la noción de comercio electrónico tanto los contratos de compraventa como los de prestación de servicio llevados a cabo en línea, o bien solamente los contratos de compraventa. Por lo que respecta a los sujetos que participan en el comercio electrónico, podría haber diversas combinaciones entre las empresas, los consumidores, e incluso gobiernos y ciudadanos. ${ }^{8}$

En el seno de la Comisión de las Naciones Unidas para el Derecho Mercantil Internacional (CNUDMI) fue elaborado un novedoso instrumento de soft law en esta materia: la Ley Modelo sobre Comercio Electrónico de $1996,{ }^{9}$ que ha sido un éxito tomando en cuenta el número de países que la han seguido. ${ }^{10}$ Los redactores decidieron evitar establecer un concepto preciso de comercio electrónico y, en su lugar, optaron por incorporar la noción de intercambio electrónico de datos (EDI), definida en el artículo 2 b) como "la transmisión electrónica de información de una computadora a otra, estando estructurada la información conforme a alguna norma técnica convenida al efecto".

8 Por ejemplo: B2B (Business to Business) entre empresas, B2C (Business to Consumer) entre empresa y consumidor, C2C (Consumer to Consumer) entre consumidores, G2C (Government to Citizen) entre gobierno y ciudadano.

Disponible en: http://www.uncitral.org/uncitral/es/uncitral_texts/ electronic_commerce/1996Model.html. Consultado el 27 de junio de 2014.

10 Ver: http://www.uncitral.org/uncitral/es/uncitral_texts/electronic_ commerce/1996Model_status.html. Consultado el 6 de julio de 2014.
A diferencia de la Ley Modelo, la Ley colombiana 527 de $1999^{11}$ contiene una serie de definiciones. En este sentido, el artículo 2 dispone:

b) Comercio electrónico. Abarca las cuestiones suscitadas por toda relación de índole comercial, sea o no contractual, estructurada a partir de la utilización de uno o más mensajes de datos o de cualquier otro medio similar. Las relaciones de índole comercial comprenden, sin limitarse a ellas, las siguientes operaciones: toda operación comercial de suministro o intercambio de bienes o servicios; todo acuerdo de distribución; toda operación de representación o mandato comercial; todo tipo de operaciones financieras, bursátiles y de seguros; de construcción de obras; de consultoría; de ingeniería; de concesión de licencias; todo acuerdo de concesión o explotación de un servicio público; de empresa conjunta y otras formas de cooperación industrial o comercial; de transporte de mercancías o de pasajeros por vía aérea, marítima y férrea, o por carretera.

Tal y como lo destaca un autor de dicho país (Plata, 2013, p. 780)-y surge sin lugar a dudas de la frase "comprenden, sin limitarse a ellas", la lista de actividades que pueden ser realizadas a partir de la utilización de mensajes de datos es enunciativa y no taxativa.

Por otro lado, entre las legislaciones más recientes encontramos la Ley francesa n. ${ }^{\circ} 2004-575$ del 21 de junio de 2004 para la confianza en

11 Por medio de la cual se define y reglamenta el acceso y uso de los mensajes de datos, del comercio electrónico y de las firmas digitales, se establecen las entidades de certificación y se dictan otras disposiciones. Disponible en: http://www.enticconfio.gov.co/index.php/ normatividad/item/232-ley-527-de-1999.html. Consultado el 6 de julio de 2014. 
la economía digital, ${ }^{12}$ que define el comercio electrónico como "la actividad económica por la cual una persona propone o garantiza a distancia y por vía electrónica la provisión de bienes y servicios" (artículo 14, párr. 1). ${ }^{13}$ Y agrega que

Entran igualmente en el ámbito del comercio electrónico los servicios tales como aquéllos que consisten en proveer informaciones en línea, comunicaciones comerciales y herramientas de búsqueda, de acceso y de recuperación de datos, de acceso a una red de comunicación o de alojamiento de informaciones, incluso cuando no son pagados por quienes los reciben (artículo 14, párr. 2).

La doctrina ha definido el comercio electrónico de manera general, indicando que "incluye toda transacción realizada por medios electrónicos" (Hocsman, 2012, p. 364). Y también, en forma más descriptiva, como

aquella modalidad de comercio en la que la mediación entre la oferta y la demanda y el perfeccionamiento de las transacciones entre ellas se realiza a través de medios digitales de comunicación, ya sea por redes abiertas o cerradas, en un mercado virtual que no posee límites geográficos (fronteras) ni temporales y no tiene una ubicación determinada, porque se encuentra en el ciberespacio (Feldstein y Scotti, 2007, p. 63) o, agregando la referencia a un "sistema global", como

el conjunto de transacciones comerciales y financieras realizadas por medios electrónicos o digitales de comunicación, ya sea por redes abiertas o cerradas, incluyendo texto, sonido e imagen, que se despliega dentro de un sistema global utilizando redes informáticas y muy especialmente Internet, el cual permite crear el mercado electrónico virtual, sin límites geográficos ni temporales, operador por ordenador y a distancia, de productos, de bienes, de servicios, de tecnologías, entre otros (Scotti, 2012, p. 54).

Desde nuestra perspectiva, el comercio electrónico es aquél que utiliza las TIc como medio para la celebración y el cumplimiento de un contrato o tan solo para la celebración del mismo (Albornoz, 2013a, pp. 365-366). ${ }^{14}$ En el primer supuesto, estamos hablando de comercio electrónico directo, mientras que en el segundo, donde el pago y/o la entrega de la cosa o la prestación del servicio pueden tener lugar en el mundo real, fuera del ambiente electrónico, nos referimos al comercio electrónico indirecto. Todas estas transacciones pueden realizarse tanto en redes abiertas o cerradas, como vía Internet o mediante el uso de teléfonos móviles u otros dispositivos inalámbricos tales como tabletas. Igualmente, quedan comprendidos en el concepto los contratos de compraventa y los contratos de prestación de servicios, sean estos

14 El enfocarnos en dos momentos críticos de la vida del contrato celebración y cumplimiento- nos permite abrazar tanto la etapa de publicidad y negociación, previas a la estricta celebración del contrato, como las transferencias electrónicas mediante las cuales una de las partes cumple su prestación e incluso el servicio de postventa. 
nacionales o internacionales, con o sin la intervención de un gobierno como parte, llevados a cabo entre empresas (B2B), entre consumidores (C2C), entre una empresa y un consumidor (B2C), o incluso entre el gobierno y un ciudadano (G2C). ${ }^{15}$

Sin embargo, no por ello pasamos por alto que en los últimos años se viene gestando una tendencia a la integración del espacio físico y el espacio virtual que podría derivar en la aparición de ciertas zonas grises en las fronteras del concepto de comercio electrónico (Rigby, 2011, pp. 65-76). Por ejemplo: si un cliente que comienza la compra de un producto buscándolo y comparando precios por Internet y finalmente se desplaza físicamente para adquirirlo en una tienda, esta operación ¿podría en un futuro cercano ser considerada como comercio electrónico? No obstante lo anterior, hoy por hoy conserva total vigencia el concepto que hemos propuesto líneas arriba.

El comercio electrónico así entendido, enmarcado en el contexto de la economía digital, cumple una función relevante en relación con el desarrollo.

\section{B. Función del comercio electrónico con respecto al desarrollo}

Para indagar acerca de cuál es la función del comercio electrónico con respecto al desarroIlo, es conveniente puntualizar qué entendemos por desarrollo. Se trata del crecimiento

15 Ver supra, nota 8. económico, ${ }^{16}$ es decir, una mayor producción de bienes y/o servicios con una inversión dada (Urquidi, 2002, p. 33). Sin embargo, la concepción amplia de desarrollo a la que nos adherimos implica que este sea no solo económico sino también social, ${ }^{17}$ además de sustentable y equitativo.

El avance en el plano económico debe traducirse en beneficios sociales para la población de un país o región en su conjunto y, mediante una distribución equitativa de la riqueza, verse reflejado en cuestiones cruciales para la vida de las personas, tales como educación, salud, salarios, jubilaciones. En cuanto al mantenimiento y continuidad del desarrollo en el tiempo, ya en 1987 la Comisión Bruntland definía el desarrollo sustentable ${ }^{18}$ como aquél que tiene lugar "sin comprometer la capacidad de las generaciones futuras para satisfacer sus propias necesidades" (Comisión Mundial sobre el Medio Ambiente y el Desarrollo, citada por Urquidi, 2002, p. 31). ${ }^{19} \mathrm{Se}$ destaca el carácter equitativo que debe tener el desarrollo sustentable, desarrollo que "deberá comprender la idea tradicionalmente aceptada

16 Obregón (2008, p. 15) señala que el desarrollo económico es un fenómeno propio de la segunda mitad del siglo XX, ya que antes el crecimiento era mínimo.

17 De hecho, hay autores que distinguen "crecimiento económico" de "desarrollo", dependiendo de si la noción se limita a los aspectos económicos o si también incorpora aspectos sociales. Ver Álvarez Gómez y Alonso González (2006, p. 2).

18 El término "sustentable" es una traducción de la palabra sustainable del inglés, que a veces también es traducida como "sostenible". Urquidi refiere que alguna vez pensó en utilizar el término "perdurable", pero que prefirió plegarse al vocabulario oficial de las Naciones Unidas y por eso adoptó "sustentable" (2002, p. 12).

19 Informe en inglés disponible en: http://www.un-documents.net/ourcommon-future.pdf. Consultado el 29 de julio de 2014. 
de contribuir, mediante políticas y programas económicos y sociales articulados, a reducir las gruesas desigualdades que se han manifestado durante el siglo XX" (Urquidi, 2002, p. 32).

Consideramos que el desarrollo así entendido puede ser impulsado por las $\operatorname{TIC}^{20}$ y por el comercio electrónico que las mismas hacen viable. En efecto, este último está llamado a desempeñar la función de promotor del desarrollo de los países de América Latina. “El comercio electrónico puede contribuir decisivamente a que las economías en desarrollo obtengan mayores beneficios del comercio" (Organización Mundial del Comercio [омs] 2013, p. 1).

Mediante la implementación de las tıc en el ámbito empresarial es posible optimizar los procesos productivos, ${ }^{21}$ particularmente en microempresas y pequeñas y medianas empresas, ${ }^{22}$ que son las estructuras productivas que actualmente tienen menos acceso a las TIC, $^{23}$ e incentivar

20 La industria de las TIC comprende las actividades de empresas que fabrican productos electrónicos tales como PC y sus componentes, que desarrollan aplicaciones de software, que prestan servicios de tecnología de la información, bases de datos y almacenamiento de datos, y distintos tipos de soluciones en paquete o ad hoc para las empresas - para gestionar contenidos institucionales, relaciones con los clientes y/o la cadena de suministro- (Ciravegna, 2012, p. $9)$. La industria de las TIC se constituye "en un motor del crecimiento económico de países de menores ingresos" (Cepal, 2013, p. 60).

21 El impacto económico de las TIC, será mayor si se actúa simultáneamente en los factores de oferta, a saber: infraestructura de telecomunicaciones e industria de las TIC, y en los factores de demanda - aumento de la digitalización del sector productivo- de la economía digital (Cepal, 2013, p. 50).

Las microempresas suelen tener de 1 a 5 (o de 1 a 10) empleados, en tanto que las pequeñas y medianas empresas (Pyme) suelen tener entre 5 (o 10) y 25 trabajadores. Cuando el número de empleados excede los 25 se puede hablar de grandes empresas (Conferencia de las Naciones Unidas sobre Comercio y Desarrollo (UNCTAD), 2010, p. 9).

"Al interior de cada país, existen brechas de productividad muy elevadas entre las microempresas y las pymes, y las grandes la exportación de bienes y servicios. Asimismo, las herramientas electrónicas permiten comercializarlos de manera veloz y efectiva, con un alcance geográfico hasta hace poco tiempo inimaginable. La industria de las TIC "tiene efectos de derrame sobre los demás sectores de la economía" (Cepal, 2013, p. 60). Así, es factible aumentar el nivel de ingresos de las empresas, fortaleciendo a su vez la economía de los países de la región. En esta misma línea de acción, resulta apropiado estimular las transacciones electrónicas con otras regiones del mundo y entre países latinoamericanos; es decir, promover el comercio electrónico interregional e intrarregional.

Un factor que ha venido resultando clave para el crecimiento del comercio electrónico en América Latina es la amplia difusión alcanzada por la telefonía celular y el acceso a Internet mediante dispositivos móviles. En efecto, “los teléfonos móviles tienen un papel cada vez mayor en la expansión del comercio electrónico en los países en desarrollo, especialmente entre los usuarios que no disponen de conexión mediante terminales" (омс, 2013, p. 5). Y dado que las TIC evolucionan permanentemente, los servicios móviles serán cada vez más avanzados, lo que abrirá un sinnúmero de posibilidades de comercio electrónico, especialmente en los países en vías de desarrollo (omc, 2013, p. 6), y tendrá un impacto en el desarrollo sustentable y equitativo de los países en cuestión.

empresas[,] que reflejan grandes diferencias en capacidades, tecnología, internacionalización y salarios" (Cepal, 2013, p. 71). 
Creemos que la participación de las microempresas y de las Pyme en el comercio electrónico es crucial en los países en desarrollo y que tales empresas "pueden beneficiarse considerablemente de la telefonía móvil, Internet y otros tipos de tecnologías de la información y la comunicación (TIC) en sus actividades comerciales cotidianas" (omc, 2013, p. 1). Sin embargo, se ha notado con preocupación que por falta de confianza (omc, 2013, p. 10), de capacidad técnica o de suficiente sensibilidad acerca de la utilidad de invertir en TIc, las Pyme de dichos países no siempre emplean las herramientas tecnológicas disponibles en todo su potencial, en el ámbito del comercio electrónico (omc, 2013, p. 11).

El impulso al comercio electrónico de bienes manufacturados y de servicios, especialmente de servicios directamente relacionados con la industria de las $\mathrm{TIC}^{24}$ habrá de contribuir a reducir la tradicional dependencia de los países latinoamericanos con respecto a la exportación de materias primas, que comporta la explotación de los recursos naturales. ${ }^{25}$ Adicionalmente, y para aprovechar que "la deslocalización de la industria de software avanza hacia la consolidación de un modelo de oferta global de servicios" (Cepal, 2013, p. 67), se ha sugerido que sería positivo que los países de la región se concentraran en el desarrollo de áreas o clusters de provisión de servicios de las TIC exportables al

24 Ver supra, nota 20.

25 Dicha dependencia implica, a su vez, que la posibilidad de exportar esté sujeta a los vaivenes del medioambiente, tema delicado si los hay en épocas de cambio climático como la que vivimos en la actualidad. Ver Ciravegna (2012, p. 130). resto del mundo. ${ }^{26}$ Un dato relevante a tener en cuenta en este sentido es el hecho de que en América Latina subsiste aún una clase media con acceso a educación y a herramientas tecnológicas, susceptible de convertirse en mano de obra para los proveedores de servicios de las TIC (Ciravegna, 2012, p. 130), lo que contribuiría a mejorar la tasa global de empleo.

El comercio electrónico facilita que los vendedores de bienes y prestadores de servicios, con mínimo esfuerzo y costo, los ofrezcan a potenciales clientes localizados en cualquier punto del mundo, los vendan e incluso -cuando la naturaleza del bien o servicio lo permite- los entreguen y cobren en línea. De esta manera, se multiplica exponencialmente el universo de clientes, lo que puede incrementar las ganancias de grandes pero también de pequeños y medianos empresarios, convirtiéndose así el comercio electrónico en un factor positivo para impulsar el desarrollo de un país y de una región.

En síntesis, el comercio electrónico desempeña la función de promotor con respecto al desarrollo de los países de América Latina.

26 Como lo ha venido haciendo Costa Rica. Ver, sobre el interesante caso de este país, Ciravegna (2012, p. 9). En este contexto, nuestra región puede volverse un punto importante de localización de la industria de software, como lo son India, China y Europa oriental. Este proceso ha comenzado gradualmente durante la década de 1990 (Cepal, 2013, pp. 67 y 69$)$. 


\section{ODR COMO HERRAMIENTA PARA FORTALECER LA SEGURIDAD JURÍDICA EN EL AMBIENTE ELECTRÓNICO}

Dadas las características propias del ambiente electrónico, ${ }^{27}$ es natural que allí surja una falta de confianza por parte de los usuarios (Albornoz, 2013a, pp. 363-386). Esto implica la necesidad de fortalecer la seguridad jurídica $(A)$. Consideramos pertinente detenernos en el concepto de ODR y examinar su función con referencia a la seguridad jurídica en el entorno donde se lleva a cabo la contratación electrónica (B).

\section{A. La seguridad jurídica en el ambiente electrónico}

El comercio electrónico se desenvuelve principalmente en un entorno virtual, aunque algunos actos propios del comercio electrónico indirecto tengan lugar en el ambiente offline. Entre los múltiples intentos de conceptualizar la seguridad jurídica ${ }^{28}$ que han sido concebidos para el mundo real, hemos decidido retener uno emanado de la jurisprudencia del Tribunal Constitucional español. La sentencia 271/1981 del referido tribunal ${ }^{29}$ indica algunos "elementos integrantes" de la seguridad jurídica: "es suma de certeza y legalidad, jerarquía y publicidad normativa, irretroactividad de lo no favorable,

27 Desmaterialización, desocialización y desjudicialización. Ver Schultz (2005, p. 197)

28 Ver, por ejemplo: Radbruch (2000, p. 40), García Máynez (1986, pp. 477-478) y Rojas Amandi (2009, pp. 337-338).

29 Citada por García-Escudero, (2010, p. 1). [e] interdicción de la arbitrariedad" (sentencia 271/1981 citada por García-Escudero, 2010, p. 18). Por consiguiente, "la seguridad jurídica es la suma de estos principios, equilibrada de tal suerte que permita promover, en el orden jurídico, la justicia y la igualdad, en libertad".

Para el ciberespacio nos sirve también esta noción de seguridad jurídica, solo que no hay que perder de vista la singular importancia que allí adquieren las técnicas flexibles de regulación (González Martín, 2001, p. 397), especialmente en materia de contratación electrónica. Asimismo, consideramos que en la actualidad el nivel de seguridad jurídica que ha alcanzado el ambiente en línea (online), aún con loables avances,$^{30}$ todavía es menor o más frágil que el que -incluso reconociendo ciertas deficiencias- puede existir en el entorno offline. ${ }^{31}$ Por ello, tomando en cuenta además que el ambiente electrónico es novedoso y que, dada su vertiginosa y continua evolución seguirá siéndolo, creemos que es indispensable fortalecer o apuntalar la seguridad jurídica existente en su seno. Esto permitirá, por un lado, mejorar los niveles de certeza y previsibilidad así como, por otro lado, mantener un estado de alerta permanente al respecto, a efectos de evitar que la implementación de nuevas tic sea capaz de debilitar la seguridad jurídica en línea.

30 Como la firma electrónica, el empleo del protocolo seguro de transferencia de hipertexto https o los sellos de confianza.

31

En cuanto a la cuestión específica del tratamiento de datos personales en ámbito del cómputo en la nube, se recomienda ver Casasola Robles, Maqueo Ramírez, Molina Rodríguez, Moreno González y Recio Gayo (2014). 
Es sabido que el principio de autonomía de la voluntad goza de una amplia aceptación en materia de contratación internacional (Symeonides, 2014, p. 114; Albornoz, 2009, p. 632) y, por consiguiente, esto también ocurre cuando aquellos contratos son electrónicos. Las partes que celebran entre sí un contrato internacional echando mano de las tic tienen la facultad de pactar cuál será el tribunal competente para resolver eventuales controversias derivadas del contrato, así como cuál será el derecho aplicable a dicho contrato. Pero, claro está, las partes no están obligadas a elegir tribunal y normas aplicables. Y cuando no lo hacen, entran en juego las reglas subsidiarias - es decir, para casos de ausencia de elección válidamente efectuadaprevistas por el derecho internacional privado de cada país, sea este de fuente internacional o autónoma.

Llegados a este punto, es factible que se presenten dificultades en la tarea de identificar tanto el tribunal competente como el derecho aplicable, lo que redundaría en confusión e incertidumbre para las partes; es decir, habría un déficit de seguridad jurídica. ¿Por qué podría haber problemas? Porque las normas de jurisdicción internacional y las normas de derecho aplicable existentes llamadas a regir el caso son, en la mayoría de los supuestos, anteriores al surgimiento y expansión del comercio electrónico. ${ }^{32}$ Entonces, contienen puntos de conexión

32 "Los principios jurídicos tradicionales son aplicables a la mayoría de las controversias de comercio electrónico. No obstante, la operación del comercio electrónico en el ciberespacio da lugar a nuevas circunstancias a las cuales los juristas no pueden aplicar de inmediato, sin inconvenientes, normas jurídicas establecidas". Davidson (2009, p. 183). Traducción de las autoras. que no han contemplado -ni han podido contemplar- la nueva realidad generada por las TIC. ¿Qué se debe entender, por ejemplo, por "lugar de celebración" o "lugar de cumplimiento" de un contrato internacional electrónico, o por "lugar de ubicación de la cosa" que se halla en el ciberespacio? ¿Tiene alguna importancia a estos efectos el país donde se localizan los servidores que han estado vinculados con el contrato? ¿Y si hay servidores espejo? ¿Es relevante el país o los países desde el cual o desde los cuales se accede a cierta página web?

¿Cómo superar estas dificultades? Una posibilidad consistiría en evaluar la necesidad de contar con nuevas normas y, en su caso, dictarlas para que entren en vigor cuanto antes. ${ }^{33}$ Otra solución que demandaría menos tiempo y sería más realista ${ }^{34}$ es la de utilizar las normas de jurisdicción internacional y de derecho aplicable existentes haciendo una calificación amplia de los puntos de conexión de las mismas, siempre que el ordenamiento jurídico del foro ponga a disposición del juez herramientas suficientes para llevar a cabo dicha calificación. También se puede prevenir la caída en este tipo de incertidumbre promoviendo el ejercicio de la autonomía de la voluntad de los contratantes. Y continuando en la vía del ejercicio de la autonomía de la voluntad de las partes, cabe explorar la

33 Se trataría de elaborar normas de conflicto o, mejor aún, normas materiales diseñadas especialmente para Internet (Calvo Caravaca y Carrascosa González, 2001, p. 15).

34 "La alternativa tradicional, es decir, la aplicación del DIPr. de cada Estado, constituye, así, la vía real que lleva a solucionar las cuestiones legales que el uso de Internet ha hecho surgir. Ello es así porque..., en realidad, los problemas jurídicos que plantea Internet no son nuevos" (Calvo Caravaca y Carrascosa González, 2001, pp. 22-23). 
factibilidad del recurso a mecanismos alternativos de solución de controversias en línea. ${ }^{35}$

\section{B. Concepto de ODR y su función con respecto a la seguridad jurídica}

“El ODR tiene cualidades adquiridas del ambiente en línea, pero también tiene rasgos adquiridos del ADR" (Katsh y Rifkin, 2001, p. 19). Efectivamente, "ha visto la luz como una adaptación de la Solución Alternativa de Controversias" (KaIlel, 2008, p. 345). Los métodos alternativos de solución de controversias (MASC) o mecanismos de Alternative Dispute Resolution (ADR) han sido exitosos en el mundo offline como una alternativa de carácter privado frente a los tribunales estatales. En América Latina estamos muy familiarizados con la sobrecarga de expedientes que agobia a los tribunales estatales de nuestros países, con el formalismo procesal, el largo período de tiempo que puede transcurrir hasta lograr obtener una sentencia, así como con los costos económicos y también emocionales de un juicio ante los tribunales oficiales (Albornoz y González Martín, 2012, p. 43). En la práctica de la resolución de diferendos, los diversos MASC -principalmente la negociación, la conciliación, la mediación y el arbitraje- han demostrado ser modos efectivos de sortear los referidos inconvenientes de los procedimientos judiciales de una forma flexible, veloz y que implica soportar costos menores.
El ODR es el resultado de la combinación de los MASC con las TIC. ${ }^{36}$ Por eso, aunque es muy positivo que los tribunales del Estado estén poco a poco incorporando a sus procedimientos ciertos avances tecnológicos ${ }^{37}$-de hecho, se los puede denominar "cibertribunales"-, 38 entendemos que en principio tales procedimientos no quedan comprendidos en la noción estricta de ODR. En otras palabras, consideramos que no constituyen una alternativa real al litigio tradicional ante los tribunales nacionales (Albornoz y González Martín, 2012, p. 44).

En la literatura se advierte la existencia de distintos enfoques acerca del ODR. De manera general, consiste en "cualquier método por medio del cual las partes intentan resolver controversias en línea" (Cole y Blanklei, 2006, p. 193). Por un lado, puede vérselo como una "simple transposición de los tradicionales MASc [al ambiente] en línea[,] sin diferencias sustanciales con respecto a sus contrapartes tradicionales[,] excepto [el hecho de] ser más conveniente y efectivo" (Tang, 2009, p. 152). Por cierto, algunos de los mecanismos de solución de diferendos diseñados para cubrir las necesidades de los usuarios de Internet "no son completamente nuevos desde una perspectiva jurídica” (Rule, 2002, p. 61).

36 Tal como lo destaca la doctrina, el ODR implica procesos de ADR "en gran medida asistidos por la velocidad y conveniencia de las TIC y de internet, que son especialmente apropiados para las necesidades del e-commerce" (Cortés 2011, p. 2). Traducción de las autoras.

37 Por ejemplo, autorizar que ciertas audiencias se lleven a cabo por videoconferencia, o emplear medios de comunicación electrónicos para notificar sus resoluciones a las partes, o al menos permitirles consultarlas en línea.

38 Los cibertribunales generalmente se estudian por separado de los métodos de ODR. Ver Montesinos García (2007, p. 41).

35 Ver Albornoz y González Martín (2012, pp. 39-61). 
Por otro lado, se concibe el oDR como

una importante herramienta nueva, un sistema nuevo, una nueva manera de hacer negocios que es más eficiente, más eficaz en función de los costos y mucho más flexible que los enfoques tradicionales... Combina la eficiencia de la solución alternativa de controversias con el poder de Internet[,] para ahorrarles a las empresas tiempo, dinero y frustraciones (Rule, 2002, p. 3). ${ }^{39}$

Además, es lógico que exista también un grupo de autores para quienes el ODR comprende no solo los MASc sino también los cibertribunales. Es decir, que adoptan un concepto amplio o extensivo de oDR. Y lo justifican explicando que "el ODR es una reacción ante las limitaciones del mundo offline y no estrictamente ante los tribunales" (Kaufmann-Kohler y Shultz, 2004, p. 7).

Estimamos apropiado hacer algunas precisiones. En primer lugar, que los métodos o mecanismos de ODR, al igual que las TIC y los requerimientos de las partes en conflicto, están permanentemente sujetos al cambio (Albornoz y González Martín, 2012, p. 46). Aunque algunos de dichos métodos toman como modelo inmediato un mecanismo tradicional de ADR -mediación o arbitraje en línea, por ejemplo-, otros tienen un mayor componente de novedad tecnológica -como la negociación automatizada, por ejemplo-. Por consiguiente, no existe una correspondencia total entre los mecanismos alternativos de solución de controversias imple-

39 También se ha afirmado que el ODR toma ciertos aspectos prestados del ADR; pero que en el futuro también el ADR tomará prestado del ODR (Katsh y Rifkin, 2001, p. 24). mentados en forma presencial o los llevados a cabo en línea (Vilalta Nicuesa, 2013, p. 42). ${ }^{40}$ En segundo lugar, hay que tener en cuenta que los mecanismos de oDR pueden ser utilizados para solucionar controversias originadas tanto en el mundo offline como en el mundo online, sean estas internas o internacionales (Vilalta Nicuesa, 2013, p. 45), y de mayor o menor cuantía (Cortés, 2011, p. 2). Destacamos no obstante que el oDR resulta ideal para lidiar con disputas originadas en línea -ya que serán estudiadas y resueltas en el mismo ambiente virtual en que nació la relación contractual entre las partes-y que presentan elementos de extranjería, especialmente si los contratantes están domiciliados en diferentes países y el monto en disputa no justifica afrontar el costo de viajes y asistencia jurídica en el extranjero (Cortés, 2011, p. 2). ${ }^{41}$ Finalmente, cabe señalar que una pregunta que aún no ha podido ser resuelta con exactitud por la doctrina es la de qué tanta actividad procesal tiene que realizarse en línea para que un determinado proceso sea considerado de oDR. Entendemos que una parte sustancial de los actos procesales ha de realizarse en línea, quedando la actividad procesal en el mundo real como algo de carácter excepcional (Albornoz y González Martín, 2012, p. 48). ${ }^{42}$

40 Esta autora agrega que las modalidades en línea "tienen un ámbito de aplicación mayor, al aprovechar el entorno que ofrece Internet" y que "Las TIC ... facilitan la generación de nuevas formas de gestión y estrategias de resolución de controversias que no encuentran correspondencia en los ADR."

41 De hecho, hasta ahora el ODR ha sido objeto de una buena recepción en el ámbito del comercio electrónico, dada la frecuente naturaleza internacional de las operaciones (González Granda, 2008, p. 3).

42 En este sentido, ver Montesinos García (2007, p. 42). 
Confianza y seguridad jurídica van de la mano. Dado que el oDR estimula la confianza en el comercio electrónico, tiene la función de contribuir a incrementar los niveles de seguridad jurídica en el contexto de las transacciones en línea. Por cierto, la utilización de oDR para la resolución de conflictos surgidos de transacciones electrónicas es un componente esencial de la arquitectura de confianza que es propicio crear (Albornoz, 2013, p. 383). De este modo, generando un impacto en el comercio electrónico y en la economía digital, el ODR tiene todo el potencial para convertirse también en un motor del desarrollo de los países menos favorecidos (Albornoz, 2013b, p. 5).

Los mecanismos de ODR, aplicados a la solución de controversias derivadas del comercio electrónico, permiten que los contratantes tengan la certidumbre de que, por más que la cuantía de su reclamo sea baja, pueden dar curso a un procedimiento extrajudicial confiable, económico y expedito, en el mismo ambiente en línea en el cual celebraron su transacción. Así, indirectamente, fortaleciendo la seguridad jurídica en el comercio electrónico, el ODR puede incidir de forma positiva en el desarrollo de un país. Adicionalmente, por su lado, y como se ha visto más arriba, un comercio electrónico floreciente impulsará el crecimiento económico y el desarrollo social de la población. El comercio electrónico será relevante para el desarrollo, inclusive si no se recurre de manera masiva a mecanismos de ODR; pero creemos que si se logra la expansión de la solución de controversias en línea, tal relevancia sería aún mayor.
En suma, consideramos que en América Latina los mecanismos de oDR se perfilan como una herramienta útil para apuntalar la seguridad jurídica en el ambiente virtual donde se desenvuelve el comercio electrónico y -por añadidura-como un elemento que puede favorecer el desarrollo de la población de nuestra región.

\section{CONCLUSIONES}

1. Las TIC, el comercio electrónico que ellas permiten, y el oDR que estimula la confianza en el comercio electrónico, pueden coadyuvar al desarrollo de los pueblos latinoamericanos.

2. El comercio electrónico tiene amplias posibilidades de crecimiento en América Latina y promoverlo contribuirá al desarrollo de la región.

3. El comercio electrónico es aquél que utiliza las TIC como medio para la celebración y el cumplimiento de un contrato o tan solo para la celebración del mismo.

4. El desarrollo que se requiere impulsar no solo debe ser económico sino también social, sustentable y equitativo.

5. Las microempresas y las Pyme latinoamericanas pueden obtener grandes beneficios a través de una mayor participación en el comercio electrónico.

6. La seguridad jurídica es la suma de los principios de certeza y legalidad, jerarquía y publicidad normativa, irretroactividad de lo no favo- 
rable, interdicción de lo arbitrario, equilibrada de manera tal que permita promover la justicia y la igualdad, en libertad, en el orden jurídico.

7. Es indispensable fortalecer la seguridad jurídica en el ambiente virtual.

8. Dado que el oDR estimula la confianza en el comercio electrónico, contribuye a incrementar los niveles de seguridad jurídica en el contexto de las transacciones en línea.

9. Es necesario promover el ejercicio de la autonomía de la voluntad de las partes del contrato electrónico internacional, tanto con respecto a la jurisdicción como en relación con el derecho aplicable.

10. La mayoría de las normas subsidiarias de jurisdicción internacional y de derecho aplicable -para casos de no ejercicio válido de la autonomía- son anteriores al surgimiento y expansión del comercio electrónico y su aplicación a esta nueva realidad puede plantear dificultades.

11. Para superar las dificultades provocadas por el desajuste entre las normas subsidiarias de jurisdicción internacional y de derecho aplicable, y el comercio electrónico, sería conducente evaluar la necesidad de contar con nuevas normas y, en su caso, dictarlas. Otra posibilidad consistiría en calificar de manera amplia los puntos de conexión de las normas existentes. Estas dificultades podrían obviarse mediante el ejercicio de la autonomía de la voluntad.
12. En virtud de la autonomía de la voluntad, las partes pueden decidir resolver sus controversias mediante ODR.

13. El ODR es el resultado de la combinación de IOS MASC con las TIC.

14. En el ODR, que es útil para solucionar controversias originadas tanto en el mundo offline como en el online, nacionales o internacionales, una parte sustancial de los actos procesales ha de realizarse en línea.

15. El ODR, al aumentar la confianza en el comercio electrónico, y el comercio electrónico, al favorecer el crecimiento de la economía digital, pueden tener un impacto altamente positivo en el desarrollo de los países latinoamericanos.

\section{Referencias}

Albornoz, M. M. (2009). El derecho aplicable a los contratos internacionales en los Estados del Mercosur. Boletín Mexicano de Derecho Comparado, XLII(125), 631-666.

Albornoz, M. M. (2013a). La falta de confianza en el comercio electrónico. En M. V. Aguilar y M. A. Munive Páez (Coords.), Memorias del XXXV Seminario Anual de la Academia Mexicana de Derecho Internacional Privado y Comparado en homenaje a la Escuela Libre de Derecho por su primer centenario (págs. 363-386). Ciudad de México: Escuela Libre de Derecho y AMEDIP. 
Albornoz, M. M. (2013b). Online Dispute Resolution as a Development Booster for Latin American Countries. Documento de trabajo de la División de Estudios Jurídicos del CIDE, n. ${ }^{\circ} 63$. Ciudad de México: CIDE.

Albornoz, M. M. y González Martín, N. (Fall 2012). Feasibility Analysis of Online Dispute Resolution in Developing Countries. University of Miami Inter-American Law Review, 44(1), 39-61.

Álvarez Gómez, J. y Alonso González, Á. (2006). Nociones de crecimiento y desarrollo económico. Revista Galega de Economía, 15(2). Recuperado el 27 de julio de 2014 de usc. es: http://www.usc.es/econo/RGE/Vol15_2/ castelan/nb1c.pdf.

America Retail. (28 de junio de 2013). Latinoamérica liderará el crecimiento en e-commerce el 2014. Recuperado el 4 de julio de 2014 de: http://america-retail.com/industria-y-mercado/latinoamerica-liderara-el-crecimiento-en-e-commerce-el-2014.

Artusa, M. (6 de julio de 2014). Vivimos en dos mundos paralelos y diferentes: el online y el offline. Obtenido de Clarín: http://www.clarin. com/edicion-impresa/Vivimos-paralelos-diferentes-online-offline_0_1169883075.html.

Calvo Caravaca, A. L. y Carrascosa González, J. (2001). Conflictos de leyes y conflictos de jurisdicción en Internet. Madrid: Editorial Colex.
Casasola Robles, M., Maqueo Ramírez, M., Molina Rodríguez, M., Moreno González J. y Recio Gayo, M. (2014). La nube: nuevos paradigmas de privacidad y seguridad para un entorno innovador y competitivo. Ciudad de México: Centro de Investigación y Docencia Económicas/cloudMIDDLEtrust, S.L.

Ciravegna, L. (2012). Promoting Silicon Valleys in Latin America. Lessons from Costa Rica. Nueva York: Routledge.

Cole, S. R. y Blankley, K. M. (2006). Online Mediation: Where We Have Been, Where We Are Now and Where We Should Be. University of Toledo Law Review, 38(1), 193-212.

Comisión de las Naciones Unidas para el Derecho Mercantil Internacional. (1996). Ley modelo de la Comisión de las Naciones Unidas para el Derecho Mercantil Internacional sobre Comercio Electrónico de 1996. Obtenido de uncitral: http://www.uncitral. org/uncitral/es/uncitral_texts/electronic_ commerce/1996Model.html.

Comisión Económica para América Latina y el Caribe. (Marzo de 2013). Economía digital para el cambio estructural y la igualdad. Recuperado el 29 de julio de 2014 de Cepal: http://www.cepal.org/publicaciones/ xml/5/49395/economia_digital_para__ cambio_estryigualdad.pdf.

Comisión Mundial sobre el Medio Ambiente y el Desarrollo. (1987). Nuestro futuro común. Madrid: Alianza Editorial. 
ComScore. (Mayo de 2013). Futuro Digital Latinoamérica 2013. El Estado Actual de la Industria Digital y las Tendencias que Están Modelando el Futuro Recuperado el 4 de julio de 2014 de ComScore: https://www.comscore.com/lat/Insights/Events-and-Webinars/ Webinar/2013/2013-Latin-America-DigitalFuture-in-Focus.

Conferencia de las Naciones Unidas sobre Comercio y Desarrollo. (2010). Informe sobre la economía de la información 2010. TIC, empresas y reducción de la pobreza. Recuperado el 28 de julio de 2014 de unctad: http:// unctad.org/es/docs/ier2010_sp.pdf.

Cortés, P. (2011). Online Dispute Resolution for Consumers in the European Union. Abingdon: Routledge.

Davidson, A. (2009). The Law of Electronic Commerce. Cambridge: Cambridge University Press.

Feldstein de Cárdenas, S. L. y Scotti, L. B. (2007). La convención sobre utilización de las comunicaciones electrónicas en los contratos internacionales: un avance hacia la armonización legislativa en materia de contratación electrónica. Revista Científica de la Universidad de Ciencias Empresariales y Sociales (UCES), XI(1). Recuperado el 20 de julio de 2012 de Universidad de Ciencias Empresariales y Sociales: http://dspace.uces.edu.ar:8180/ xmlui/handle/123456789/198.
García-Escudero Márquez, P. (2010). Técnica legislativa y seguridad jurídica: ¿hacia el control constitucional de la calidad de las leyes? Cizur Menor: Civitas.

García Máynez, E. (1986). Filosofía del Derecho (Quinta ed.). Ciudad de México: Editorial Porrúa.

Gola, R. V. (2013). Droit du commerce électronique: guide pratique du e-commerce. París: Gualino-Lextenso éditions.

González Granda, P. (2008). El arbitraje en el ámbito del comercio electrónico: perspectivas de futuro en el ordenamiento español. Revista Vasca de Derecho Procesal y Arbitraje, 20(1), 1-13.

González Martín, N. (2011). Private International Law in Latin America: from Hard to Soft Law. Anuario Mexicano de Derecho Internacional, XI, 393-405. Recuperado el 10 de julio de 2014 de unam: http://biblio.juridicas. unam.mx/estrev/pdf/derint/cont/11/art/ art13.pdf

Hocsman, H. S. (2010). Los contratos electrónicos. En R. A. Etcheverry y R. Illescas Ortiz, Comercio electrónico: estructura operativa y jurídica. Buenos Aires: Hammurabi/José Luis Depalma Editor.

International Telecommunication Union. (2013). El mundo en 2013. TIC: Datos y cifras relativos a las TIC. Recuperado el 4 de julio de 2014 de itu.int: http://www.itu.int/en/ITU-D/ 
Statistics/Documents/facts/ICTFactsFigures2013-s.pdf

International Telecommunication Union. (2014). The World in 2014: ICT Facts and Figures. Obtenido de ITU: http://www.itu.int/en/ITUD/Statistics/Documents/facts/ICTFactsFigures2014-e.pdf.

Kallel, S. (2008). Online Arbitration. Journal of International Arbitration, 25(3), 345-353.

Katsh, E. y Rifkin, J. (2001). Online Dispute Resolution. Resolving Conflicts in Cyberspace. San Francisco: Jossey-Bas.

Kaufmann-Kohler, G. y Schultz, T. (2004). Online Dispute Resolution: Challenges for Contemporary Justice. The Hague: Kluwer Law International, cop.

Ley colombiana 527 de 1999, medio de la cual se define y reglamenta el acceso y uso de los mensajes de datos, del comercio electrónico y de las firmas digitales, y se establecen las entidades de certificación y se dictan otras disposiciones. (28 de agosto de 2012). Obtenido de enticconfío: http://www.enticconfio. gov.co/index.php/normatividad/item/232ley-527-de-1999.html.

Ley francesa $n .^{\circ} 2004-575$ del 21 de junio de 2004 para la confianza en la economía digital. (2004). Obtenido de Legifrance: http:// www.legifrance.gouv.fr/affichTexte.do?cidTex te=JORFTEXTO00000801164.
Montesinos García, A. (2007). Arbitraje y nuevas tecnologías. Cizur Menor: Civitas.

Obregón, C. (2008). Teorías del desarrollo económico. Ciudad de México: Pensamiento Universitario Iberoamericano.

Organización Mundial del Comercio. (2013). El comercio electrónico en los países en desarrollo. Oportunidades y retos para las pequeñas y medianas empresas. Ginebra, Suiza: oms. Recuperado el 20 de julio de 2014 de wto: http://www.wto.org/spanish/res_s/ booksp_s/ecom_brochure_s.pdf.

Plata López, L. C. (2013). La protección a los consumidores en el comercio electrónico. En J. Gual, J. Villalba y C. Lima, Derecho del Consumo: problemáticas actuales. Bogotá: Universidad Santo Tomás/Grupo Editorial Ibáñez.

Programa de las Naciones Unidas para el Desarrollo. (2013). Informe sobre Desarrollo Humano 2013. El ascenso del Sur: Progreso humano en un mundo diverso. Recuperado del 7 de julio de 2014 de UNDP: http://hdr.undp. org/sites/default/files/hdr2013_es_complete.pdf.

Programa de las Naciones Unidas para el Desarrollo. (2014). Informe sobre Desarrollo Humano 2014. Sostener el Progreso Humano: reducir vulnerabilidades y construir resiliencia. Texto completo en inglés disponible en: http://hdr.undp.org/sites/default/files/ hdr14-report-en-1.pdf. 
Rabagny-Lagoa, A. (2011). Droit du commerce électronique. París: Ellipses Édition Marketing S.A.

Radbruch, G. (2000). Introducción a la Filosofía del Derecho. Ciudad de México: Fondo de Cultura Económica.

Rigby, D. (Diciembre de 2011). The Future of Shopping. Harvard Business Review, pp. 65-76. Recuperado el 1 de julio de 2014 de Harvard Business Review: http://hbr. org/2011/12/the-future-of-shopping/ar/1.

Rojas Amandi, V. M. (2009). Filosofía del Derecho (Segunda ed.). Ciudad de México: Oxford University Press.

Rule, C. (2002). Online Dispute Resolution for Business: B2B, E-Commerce, Consumer, Employment, Insurance, and Other Commercial Conflicts. San Francisco: Jossey-Bass.

Scotti, L. B. (2012). Contratos electrónicos: un estudio desde el Derecho Internacional Privado argentino. Buenos Aires: Eudeba.

Schultz, T. (2005). Réguler le commerce électronique par la résolution des litiges en ligne. Une approche critique. Bruxelles: Bruylant/ Paris: LGDJ.

Statista. (2014). Mobile penetration rate in Latin America from 2007 to 2015. Recuperado el 4 de julio de 2014 de statista: http://www. statista.com/statistics/218141/mobile-penetration-rate-in-latin-america-since-2007/.

Symeonides, S. (2014). Codifying Choice of Law around the World. An International Comparative Analysis. Oxford: Oxford University Press.

Tang, Z. S. (2009). Electronic Consumer Contracts in the Conflict of Laws. Oxford: Hart Publishing.

Tapscott, D. (1997). The Digital Economy: Promise and Peril in the Age of Networked Intelligence. Nueva York: McGraw-Hill.

UNdata. (2013). Composition of macro geographical (continental) regions, geographical sub-regions, and selected economic and other groupings. Recuperado el 6 de Julio de 2014 de: http://unstats.un.org/unsd/ methods/m49/m49regin.htm.

Urquidi, V. L. (2002). Los desafíos del desarrollo sustentable en la región latinoamericana. Serie Cuadernos de Trabajo Número 5. Ciudad de México: Centro de Estudios Demográficos y de Desarrollo Urbano.

Vilalta Nicuesa, A. E. (2013). Mediación y arbitraje electrónicos. Cizur Menor: Thomson Reuters Aranzadi. 